EPJ Web of Conferences 78, 03002 (2014)

DOI: 10.1051 /epjconf / 20147803002

(C) Owned by the authors, published by EDP Sciences, 2014

\title{
Wigner and the groups in classifying elementary particles and nuclear states
}

J. Cseh

Institute for Nuclear Research, Hungarian Academy of Sciences, Debrecen, Pf. 51, Hungary-4001

\begin{abstract}
The application of group-representations in nuclear and particle physics, as initiated by Wigner [1], is considered. The general classification of the elementary particles according to the representations of the inhomogenous Lorentz-group [2] is recalled. The role of the U(4) group is discussed, first in relation with the spin-isospin supermultiplets [3] both in nuclear and in particle physics, then as a dynamical group of the two-body systems in both disciplines. Finally the classification of the nuclear states is mentioned both for the single shell, and for the multi-shell problems.
\end{abstract}

\section{Introduction}

Wigner did a pioneer work in the application of group theory in physics. An important milestone was the publication of his famous textbook [1]. As its title and publisher indicate it was published when he was working in Germany, however, it is worth mentioning at this conference that he wrote this book mainly in Hungary, when he came home for vacation. Further important discoveries are (among others) the supermultiplet theory [3], and the representations of the inhomogeneous Lorentz-group [2]. In this contribution first we recall some features of the classification scheme of the elementary particles [2]. Then we consider a few consequences of the U(4) supermultiplet theory both in nuclear and in particle physics, and mention briefly a later application of the U(4) symmetry, related to the space degrees of freedom, in both disciplines. Finally the classification of the nuclear states, and fundamental models of nuclear structure is described.

\section{Classification of elementary particles}

The inhomogeneous Lorentz-group, or Poincaré-group contains the $\mathrm{SO}(3,1)$ Lorentz-transformations, as well as the translation of the 4 dimensional space-time. It is a basic assumption that the physical laws are invariant under the Poincaré-transformations, therefore, a particle (fundamental or composite) transforms according to an irreducible representation (irrep) of the Poincaré group. It implies that a particle has definite mass and spin (these quantities are the representation labels), and that to each particle is associated an antiparticle of the same mass and spin (see e.g [4], and references therein). The eigenvalue equations of the invariant operators of the Poincaré-group (for specific representations) provide us with basic wave-equations, as indicated in Table 1 [5].

This essential paper by Wigner has some interesting historical aspects, too. First it was not accepted for publication. Later the series of international Wigner-symposium (still going on) started as a 
Table 1. Reperesentations of the Poincaré group and basic wave equations.

\begin{tabular}{|c|c|c|}
\hline Mass & Spin & Equation \\
\hline finite & 0 & Klein-Gordan \\
finite & $\frac{1}{2}$ & Dirac \\
0 & $\frac{1}{2}$ & Weyl \\
0 & 1 & Maxwell \\
\hline
\end{tabular}

memorial meeting to celebrate its appearance. It is also remarkable that Wigner returned to this topic with some delay, and published further papers [6] on the geometrical interpretation with a co-author.

The classification of basic equations of Table 1 is a nice illustration of the important role of symmetries, as discussed by Wigner in his Nobel-lecture [7]: the symmetries govern the natural laws in a similar way, how the natural laws govern the events: i.e. they do not determine what is going to happen (what the law is), rather they determine what can or can not happen (be a law).

\section{$3 \mathrm{U}(4)$}

Supermultiplets: In 1937 Wigner [3], and independently Hund [8] extended Heisenberg's idea of isospin [9] to a spin-isospin SU(4) symmetry. It is based on the assumption:

$$
\left[H, \Sigma_{k} s_{\mu}(k)\right]=\left[H, \Sigma_{k} t_{\mu}(k)\right]=\left[H, \Sigma_{k} s_{\mu}(k) t_{\mu}(k)\right]=0,
$$

where $H$ is the Hamiltonian, $s_{\mu}(k)$ and $t_{\mu}(k)$ are the spin and isospin components of nucleon $k$; i.e. the energy operator commutes with the generators of the SU(4) group. This model is called supermultiplet-theory (since it combines the spin and isospin multiplets).

In nuclear physics one of the important consequences of the supermultiplet theory is the selection rule for the $\beta$-decay. Since the operator of the Gamow-Teller transition is proportional to the generator $\Sigma_{k} s_{\mu}(k) t_{\mu}(k)$, it follows that Gamow-Teller $\beta$-decay can not take place between states belonging to different $\mathrm{SU}(4)$ representations.

Further support for this symmetry comes from the study of the binding energy of nuclei. Based on the liquid drop model von Weizsacker invented a simple, yet surprisingly accurate formula [10] for its description, containing a volume, surface, Coulomb, and symmetry energy term (the last part is a consequence of the Pauli-principle, by maximizing the availability of the lowest quantum states). Self-conjugate nuclei $(N=Z)$, however, turned out to be unusually tightly bound, so an extra term is needed for the description of their binding energy. This effect was discovered by Wigner [11], and the extra energy is called Wigner-energy. Both the original [11], and the more recent [12] explanation of the Wigner energy is based on the SU(4) symmetry.

A further application of the SU(4) symmetry is related to the shell model of atomic nuclei. In the L-S coupled description it accounts for the distribution of the consequences of antisymmetrization between the space and internal degrees of freedom, thus providing a basis also for calculations with strong symmetry-breaking terms [13]. In the present-day no-core (and ab initio) shell models [14] the L-S-coupled scheme seems to be even more important, than in the traditional approach.

In particle physics the supermultiplet theory predicts e.g. a ratio of the proton and neutron magnetic moment of $-3 / 2$ [15], which should be compared to the experimental value of -1.46 .

The muon-capture can also be used for testing the supermultiplet theory (see [16] and references therein). In particular a set of relations between the transition matrix elements can be deduced from the symmetry, carrying important information on the hadron-lepton coupling. 
The combined evidences of the mass formula, the $f t$-values of the SU(4)-forbidden transitions, the nucleon magnetic moment and the muon-capture rate provide us with a convincing proof that SU(4) is a relevant approximate symmetry.

The acceptance of Wigner's supermultiplet theory was relatively slow. The main reason is that it was written too early, when the knowledge on the nuclear forces, and weak interaction was not yet sufficient. "In some prophetic way they were anticipated by Wigner" [16].

In addition to its direct application the supermultiplet theory played an important role also by inspiring further symmetries in nuclear and particle physics.

Spectrumgeneration: The U(4) group proved to be relevant also in relation with the space degrees of freedom. The rotational-vibrational motion of a two-body system can be described by an algebraic model (of U(4) group-structure), called vibron model [17]. (A simple example may help to understand this statement: the harmonic oscillator, as one possible example for the interaction of the two-body system, has a U(3) symmetry-algebra, and in order to generate the spectrum one needs an extra degree of freedom.) The spectrum is generated in this treatment by a finite $(N)$ number of bosons, which interact with each other. The model has two dynamical symmetries (limiting cases), which provide us with analytical solutions of the eigenvalue-problem, and with complete sets of basis states. They are labeled by the algebra-chains:

$$
U(4) \supset U(3) \supset O(3), \quad U(4) \supset O(4) \supset O(3) .
$$

This model is applicable both is molecular physics, as well as in nuclear and in particle physics. The most extensive applications have been carried out in relation with molecular spectra [18]. Here we recall some basic features of its applications in particle and nuclear physics. When applying it in different fields, then in addition to the basic similarities, there are important differences as well, due to the different nature of the problem. The rotation-vibration of a two-atomic molecule can be described without taking into account further degrees of freedom. A two-quark system has internal quantum numbers in addition to the space degrees of freedom of the vibron model. The nuclear two-cluster system also has internal symmetries, furthermore the clusters are composite objects, therefore, the exclusion principle may also be important. The basic features of the applications in different fields are compared in [19].

In particle physics the first applications dealt with the mesonspectrum. A meson is considered in the constituent quark model as a quark-antiquark system. In addition to the space degrees of freedom it has color $(C)$, flavor $(F)$, and spin $(S)$ quantum numbers, thus the algebra of a two-quark model is

$$
S U_{C}(3) \otimes S U_{F}(n) \otimes S U_{S}(2) \otimes U_{R}(4) .
$$

In case of multi-quark system the $\mathrm{U}_{R}(4)$ space-part may change, but otherwise the algebraic stucture is the same. The mass formula of the algebraic model, which is similar to that of the string-like models of QCD, was applied for the description of the meson spectrum in [20], while the electromagnetic decays were considered in [21]. More recently it was also applied for the description of the quarkdiquark (barion) and diquark-antidiquark (tetraquark) systems [22].

In nuclear physics the vibron model describes the relative motion of a two-cluster system. The first fully algebraic cluster model, in which not only the basis states are characterized by the irreps of some groups, but the physical operators are also expressed in terms of its generators was constructed on the phenomenologic level. It is the nuclear vibron model [23], in which the internal structure of the clusters is accounted for by the interacting boson model (of U(6) group structure) [24], and the relative motion is treated in terms of the vibron model.

In the semimicroscopic algebraic cluster model [25] the internal structure of the clusters is described by the Elliott-model, [26] thus the model of a binary configuration has an algebraic structure 
of

$$
U_{C_{1}}^{S T}(4) \otimes U_{C_{1}}(3) \otimes U_{C_{2}}^{S T}(4) \otimes U_{C_{2}}(3) \otimes U_{R}(4),
$$

an U(3)-coupled basis is used, and the Pauli-forbidden irreps are excluded from the model space. This model has been applied for the description of the cluster spectra of light nuclei [27], as well as for studying the shape isomers and the reaction channels which can populate them [28]. Extensions to the heavy nuclei [29] were considered relying on the pseudo-SU(3) [30] or [31] on the quasi-dynamical SU(3) [32] symmetries.

\section{Classification of nuclear states}

The fundamental models of nuclear structure are based on different physical pictures. The shell model indicates that the nucleus is something like a small atom, the cluster model suggests that it is similar to a molecule, while the collective model says that it is a microscopic liquid drop. Therefore, in order to understand the nuclear structure we need to study the interrelation of these models, find their common intersection, etc.

The basic connections were found in the fifties. Elliott [26] showed how the quadrupole deformation and collective rotation can be derived from the spherical shell model: the states belonging to a collective band are determined by their specific SU(3) symmetry. Wildermuth and Kanellopoulos [33] (following Perring and Skyrme [34]) established the relation between the shell and cluster models. They proved that the Hamiltonians of the two models can be rewritten into each other exactly in the harmonic oscillator approximation. This relation results in a close connection between the corresponding eigenvectors, too: the wavefunction of one model is a linear combination of those of the other, which belong to the same energy. Later on this relation was interpreted by Bayman and Bohr [35] in terms of the SU(3) symmetry. As a consequence, the cluster states are also selected from the shell model space by their specific SU(3) symmetries. This connection was established for a single major shell problem.

By incorporating the multishell excitations one can account for e.g. the electromagnetic transitions without effective charge. Algebraic models have been constructed for the description of this vertical extension in each of the three approaches. The most relevant ones from the viewpoint of the symmetry-based interrelations are as follows. The symplectic shell model has an $\mathrm{Sp}(3, \mathrm{R})$ algebraic structure [36]. The contracted symplectic model (of $\mathrm{U}_{b}(6) \otimes \mathrm{U}_{s}(3)$ ), is a collective model, obtained as the large $N$ limit of the multi major-shell symplectic model [37, 38]. The fully microscopic $[39,40]$ as well as the semimicroscopic algebraic cluster model [25] are equiped with a $\mathrm{U}_{C}(3) \otimes \mathrm{U}_{R}(3)$ basis. All these models are based on the L-S-coupled scheme, and the spin-isospin sector is described by Wigner's $\mathrm{SU}^{S T}(4)$ symmetry. The common intersection of these models is provided by the $\mathrm{U}_{x}(3) \otimes \mathrm{U}_{y}(3) \supset \mathrm{U}(3)$ dynamical symmetry [41], i.e. for the many major-shell problem this symmetry substitutes the simple SU(3). For the shell and collective models $x$ stands for the band-head (valence shell), for the cluster model it refers to the internal cluster structure. $y$ indicates in each case the major shell excitations; in the shell and collective model cases it takes place in steps of $2 \hbar \omega$, connecting oscillator shells of the same parity, while in the cluster case it is in steps of $1 \hbar \omega$, incorporating all the major shells. As a consequence the model space of the three models have a considerable overlap, but they are not identical. When the subspace of the common SU(3) irreps is equipped with an SU(3) dynamically symmetric interaction, then a complete spectrum can be obtained, which is the common intersection of the three basic structure models, i.e. it can be considered as a shell, collective, or cluster spectrum at the same time [41]. (The Wildermuth-connection between the shell and cluster models turns out to be valid also for SU(3) dynamically symmetric interactions, which is more general than that of the harmonic oscillator [42].) 


\section{Summary and conclusions}

In this contribution we have considered some applications of group-representations in classifying elementary particles and nuclear states. In particular, the exact space-time symmetry of the Poincare group serves as a general classification scheme for elementary particles. Dynamically broken unitary symmetries proved to be important in both disciplines. As an example the U(4) symmetry was discussed. It was invented by Wigner for the description of spin-isospin degrees of freedom, but it proved to be important also as a dynamical group of the rotational-vibrational motion of the two-body system. Other unitary symmetries turned out to be important both in particle and in nuclear physics. Notable examples are the SU(3) and SU(6). SU(3) was found as a symmetry group of the space sector of the shell model in nuclear physics [26], and as an internal (flavor) symmetry of the hadrons [43]. $\mathrm{SU}(6)$ is a combined spin-flavour hadron symmetry [44], and the dynamical algebra of the algebraic model for quadrupole collectivity of nuclei [24]. Unitary symmetries seem to be able to bridge the fundamental nuclear structure models, and classify the nuclear states, too. It is U(3) for a single shell problem, and $\mathrm{U}(3) \otimes \mathrm{U}(3)$ for multi-shell excitations.

These symmetries were either invented by Wigner, or they have the same nature, inasmuch they are global symmetries, described by Lie-groups. In the meantime other kind of symmetries proved to be important as well. We mention here two of them. Local symmetries, in particular local gauge invariance is the present guiding principle in the theory of fundamental interactions. The standard model, describing the electroweak and strong interaction has an $\mathrm{U}(1) \mathrm{xSU}(2) \mathrm{xSU}(3)$ local gauge symmetry. Local gauge symmetry is essential in particle physics, but not so much in nuclear physics.

Another new symmetry is the supersymmetry, transforming bosons and fermions into each other. It is described by graded Lie-groups. This seems to be relevant both in particle and in nuclear physics, though in somewhat different form [45]. In particle physics the gauge supersymmetry has important consequences: it predicts the existence of superpartner bosons or fermions of the known fermions or bosons [46]. At present we have no experimental evidence for such superpartners. In nuclear physics the dynamical supersymmetry provides a unified description of the spectra of neighbouring nuclei with even and odd mass number [47]. In this scheme the bosons are phonons of the quadrupole vibrations, and the fermions are nucleons. There is experimental evidence for the appearance of this kind of supersymmetry in nuclear spectroscopy [48]. (It was also conjectured, that similar supersymmetry may exist in relation with the dipole vibration of the clusters [49].)

In conclusion we can say that the "Gruppenpest", how the application of the group theoretical methods was called (after its invention by Wigner and others) turned out to be a funny disease. It did not hurt the physics; on the contrary: it contributed a lot to its development.

This work was supported by the OTKA (Grant No K106035).

\section{References}

[1] E P. Wigner, Gruppentheorie und Ihre Anwendung auf die Quantenmechanik der Atomspektren, Braunschweig, F. Vieweg und Sohn, 1931.

[2] E.P. Wigner, Ann. Math. 40, 149 (1939).

[3] E.P. Wigner, Phys. Rev. 51, 106 (1937).

[4] K. Huang, Quarks, Leptons and Gauge Fields, (2nd edition) World Scientific, Singapore 2008.

[5] J.P. Elliott, P.G. Dawber, Symmetry in Physics Vol. 2., MacMillen Publishers Ltd., Chippenham, 1985.

[6] Y.S. Kim, E.P. Wigner, J. Math. Phys. 28, 1175 (1987); 31, 55 (1990).

[7] E.P. Wigner, Nobel Lecture 1963. 
[8] F. Hund, Z. Phys. 105, 202 (1937).

[9] W. Heisenberg, Z. Phys. 77, 1 (1932).

[10] C.F. von Weizsacker, Z. Phys. 96, 431 (1935).

[11] E.P. Wigner, Phys. Rev. 51, 947 (1937).

[12] D.D. Warner, M.A. Bentley, P. van Isacker, Nature Phys. 2, 311 (2006).

[13] I. Talmi, Simple Models of Complex Nuclei, Harwood Academic Publisher, Chur, 1993.

[14] T. Dytrych, K.D. Sviratcheva, J.P. Draayer, C. Bahri, and J.P. Vary, J. Phys. G 35, 123101 (2008).

[15] F. Gursey, A. Pais, R.A. Radicati, Phys. Rev. Lett. 13, 299 (1964).

[16] L.A. Radicati, in Symmetry properties of nuclei, Proc 15th Solveay Conf. in Physiscs, 1970, Gordon and Beach Science Publisher, London, 113 (1971).

[17] F. Iachello, Phys. Rev. C 23, 2778 (1981);

F. Iachello, R.D. Levine, J. Chem. Phys. 77, 3046 (1982).

[18] F. Iachello, R.D. Levine, Algebraic Theory of Molecules, Oxford University, Oxford, 1995.

[19] F. Iachello, J. Cseh, G. Lévai, Heavy Ion Phys. (Act. Phys. Hung. N.S.) 1, 91 (1995).

[20] F. Iachello, N.C. Mukhopadhyay, L. Zhang, Phys. Lett. B 256, 295 (1991); Phys. Rev. D 44, 898 (1991).

[21] F. Iachello, D. Kusnezov, Phys. Lett. B 255, 493 (1991).

[22] E. Santopinto, G. Galatá, Phys. Rev. C 75, 045206 (2007); 86, 045202 (2012).

[23] H.J. Daley, F. Iachello, Ann. Phys. (N.Y.) 167, 73 (1986).

[24] F. Iachello, and A. Arima, The Interacting Boson Model, Cambridge University Press, Cambridge, 1987.

[25] J. Cseh, Phys. Lett. B 281173 (1992);

J. Cseh, G. Lévai, Ann. Phys. (NY) 230165 (1994).

[26] J.P. Elliott, Proc. Roy. Soc. A 245 128, 562 (1958).

[27] G. Lévai et al, Phys. Rev. C 46, 548 (1992); 48, 1724 (1993); 50, 2240 (1994); Nucl. Phys. A 604, 286 (1996); Phys. Lett. B 381, 1 (1996); Phys. Rev. Lett. 82, 775 (1999); Phys. Rev. C 85, 034333 (2012); 88, 014328 (2013);

P.O. Hess et al., Phys. Rev. C 74, 024319 (2006); 85, 014316 (2012); 85, 014317 (2012); 86, 034309 (2012).

[28] J. Cseh et al., J. Phys. G 18, 1419 (1992); 19, L97 (1993);

J. Darai et al., Phys. Rev. C 70, 034311 (2004); 80, 034319 (2009); 80, 034320 (2009); 84, 024302 (2011); 86, 064309 (2012).

[29] J. Cseh et. al., Phys. Lett. B 299, 205 (1993); J. Phys. G 22, L39 (1996).

[30] J.P. Draayer, Nucl. Phys. A 520 259c (1990);

C. Bahri, J.P. Draayer, S.A. Moszkowski, Phys. Rev. Lett. 682133 (1992).

[31] A. Algora et al, J. Phys. G 24, 2111 (1998); Eur. Phys. J. A 15, 449 (2002); Phys. Lett. B 639, 451 (2006).

[32] P. Rochford, D. J. Rowe, Phys. Lett. B 210, 5 (1988);

D. J. Rowe, P. Rochford, J. Repka, J. Math. Phys. 29, 572 (1988).

[33] K. Wildermuth, Th. Kanellopoulos, Nucl. Phys. 7150 (1958).

[34] J.K. Perring, T.H.R. Skyrme, Proc. Roy. Soc. A 67600 (1956).

[35] B.F. Bayman, A. Bohr Nucl. Phys. 9596 (1958/59).

[36] G. Rosensteel, D.J. Rowe, Phys. Rev. Lett. 3810 (1977); Ann. Phys. (N.Y.) 126343 (1980);

D.J. Rowe, Rep. Prog. Phys. 481419 (1985).

[37] D.J. Rowe, G. Rosensteel, Phys. Rev. C 253236 (1982). 
[38] O. Castanos, J.P. Draayer, Nucl. Phys. A 491, 349 (1989).

[39] H. Horiuchi, Prog. Theor. Phys. Suppl. 6290 (1977).

[40] K.T. Hecht, E.J. Reske, T.H. Seligman, W. Zahn, Nucl. Phys. A356 146 (1981).

[41] J. Cseh, arXiv 1404.3500 [nucl-th] (2014).

[42] J. Cseh, K. Kato, Phys. Rev. C 87067301 (2013).

[43] M. Gell-Mann, Phys. Rev. 125 128, 1067 (1962);

Y. Ne'eman, Nucl. Phys. 26, 222 (1961).

[44] F. Gursey, R.A. Radicati, Phys. Rev. Lett. 13, 173 (1964).

[45] F. Iachello, AIP Conf. Proc. 1488, 402 (2012).

[46] J. Wess, B. Zumino, Nucl. Phys. B 70, 39 (1974).

[47] F. Iachello, Phys. Rev. Lett. 44, 772 (1980).

[48] A. Frank, J. Jolie, P. Van Isacker, Symmetry in Atomic Nuclei, Springer Science, New York, 2009.

[49] G. Lévai, J. Cseh, P. Van Isacker, Eur. Phys. J. A 12, 305 (2001); J. Phys. G 34, 1729 (2007). 
